\title{
Photonic components for spacecraft fiber optic datalinks and free-space optical communications terminals
}

Ronald Logan, Davinder Basuita

Ronald T. Logan Jr., Davinder Basuita, "Photonic components for spacecraft fiber optic datalinks and free-space optical communications terminals," Proc. SPIE 11852, International Conference on Space Optics - ICSO 2020, 1185244 (11 June 2021); doi: 10.1117/12.2599639

SPIE Event: International Conference on Space Optics - ICSO 2021, 2021, Online Only 


\section{International Conference on Space Optics-ICSO 2020}

Virtual Conference

30 March-2 April 2021

Edited by Bruno Cugny, Zoran Sodnik, and Nikos Karafolas
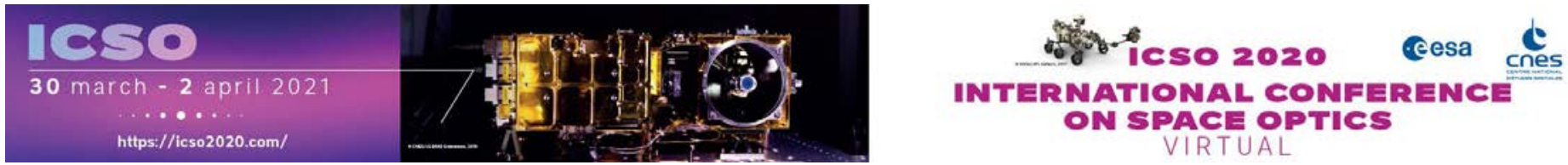

\section{Photonic components for spacecraft fiber optic datalinks and free- space optical communications terminals}

\section{Cesa issopoceatings lecnes}




\title{
Photonic Components for Spacecraft
}

Fiber Optic Datalinks and

\section{Free-Space Optical Communications Terminals}

\author{
R.T. Logan Jr.*a and Davinder Basuita ${ }^{\mathrm{b}}$ \\ a'Glenair Inc., 1211 Air Way, Glendale, CA, USA 91201; \\ ${ }^{\mathrm{b}}$ Glenair U.K. Ltd., Oakham Business Park, 40 Lower Oakham Way \\ Mansfield NG18 5BY, United Kingdom
}

\begin{abstract}
Commercial-off-the-shelf photonic components designed for datacenter or industrial applications do not typically satisfy the environmental ruggedness requirements of aerospace applications. In order to reduce costs and schedule risk for insertion of photonic components into these harsh-environment applications, we developed ruggedized fiber-optic transceiver and optical amplifier modules to support both on-board fiber-optic datalinks as well as inter-satellite free-spaceoptical (FSO) communications systems.

Parallel-optical transceiver components were developed to support data rates up to $25 \mathrm{Gbps}$ per fiber, for high-density spacecraft onboard datalinks over the $-40 \mathrm{C}$ to $+85 \mathrm{C}$ temperature range, based on a novel hermetic opto-electronic hybrid construction. These components utilize MTP 12-fiber optical and high-speed electrical connectors for ease of installation and removal and survive extremely high levels of shock and vibration. The extremely efficient heatsinking the lasers, photodiodes and integrated amplifier circuits permits operation up to $85 \mathrm{C}$ case temperature while maintaining low device junction temperatures required for high reliability.

Dense-wavelength-division-multiplexed (DWDM) transceivers were also developed to support multi-rate FSO systems from $<1 \mathrm{Gbps}$ to $12.5 \mathrm{Gbps}$ for FSO links on 60 ITU grid wavelengths. The transceivers permit user optimization of link parameters to attain highest performance in amplified systems. High-power Erbium-Ytterbium booster optical amplifiers up to 5W output power, and low-noise Erbium pre-amplifiers, in the 1540-1565 nm wavelength band, were also developed and tested in a space vacuum environment.

We performed reliability and environmental testing to demonstrate that these modules meet or exceed many of the requirements of space applications. In this presentation we will show performance characteristics and results of reliability and environmental tests for these transceiver components.
\end{abstract}

Keywords: SpaceWire, SpaceFibre, Spacecraft Datalinks, Free Space Optical (FSO) Links, Spacecraft Electronics, Spacecraft Photonics, Avionics.

\section{INTRODUCTION}

As data rates and volumes increase both on-board and between satellites, optical transmission techniques become increasingly attractive. Optical fiber interconnects between modules on a spacecraft are needed to support data rates up to $28 \mathrm{Gbps}$ or higher. Optical fiber is much lighter and smaller than copper coax of equivalent bandwidth and length, and are immune to radio-frequency (RF) interference from adjacent cables, so they require no heavy RF shielding. In a previous paper $^{1}$, we reviewed the substantial mass reductions possible by replacing copper cables with fiber optics.

*rlogan@glenair.com; phone +1 818247 6000; www.glenair.com 
Free-space optical links between spacecraft are needed to support satellite constellations, and require robust wavelengthselected transceivers with special capabilities, and both high-power and low-noise optical amplifiers. But the availability of suitable photonic components for these space applications has not been widespread, and manufacturers in the photonics industry are typically not able or willing to address the highly-specialized requirements, long design cycles, extreme environmental robustness, ultra-high reliability, vacuum operation, traceability, radiation tolerance and small, inconsistent production volumes encountered with space applications.

We have conducted a development program for aerospace-grade photonic transceivers since 2011, addressing the increasing data transmission requirements between avionics modules onboard and between spacecraft. In previous papers ${ }^{1,2,3}$, we discussed the development and test results of photonic transmitters, receivers and transceivers for aerospace applications, and highlighted the challenges with spacecraft transceiver design. In this paper we will describe the most recent results of these developments for aerospace-grade parallel optics transceivers, DWDM transceivers and optical amplifiers.

\section{PARALLEL OPTICAL TRANSCEIVERS}

Many benefits in size and mass can be obtained by moving from individual optical contacts, connectors and cabling to multiple-fiber "ribbon cable" and multi-fiber MT connectors. Multi-fiber circular D38999-style and rectangular micro-D connectors utilizing 12-fiber "MT" contacts are illustrated in Figure 1. Such a connector solution is also envisioned by the SpaceFibre standard, and requires suitably rugged parallel optical transceivers to maintain alignment to make use of all of the fibers.

Parallel-optical transceiver components were developed to support data rates from 10 Gbps to 28 Gbps per fiber, for highdensity spacecraft onboard datalinks over the $-40 \mathrm{C}$ to $+85 \mathrm{C}$ temperature range, based on a novel hermetic opto-electronic hybrid construction. These components utilize MTP 12-fiber optical and high-speed electrical connectors for ease of installation and removal and survive extremely high levels of shock and vibration. The extremely efficient heatsinking the lasers, photodiodes and integrated amplifier circuits permits operation up to $100 \mathrm{C}$ case temperature while maintaining low device junction temperatures required for high reliability.

The SpaceFibre standard provides for multi-lane "parallel optical" transceivers in addition to single-lane devices, up to eventual rates of $10 \mathrm{Gbps}$ (although only lower rates are supported the time of this writing). We developed rugged 10 Gbps parallel optical transceivers equivalent to the commercial QSFP+ standard, but ruggedized by utilizing a hermetically-sealed hybrid optoelectronic microcircuit assembly and active optical alignment process, shown in Figure 2. This construction provides for enhanced optical output power per lane of up to $+3 \mathrm{dBm}$ at $850 \mathrm{~nm}$ and sensitivity of -12 $\mathrm{dBm}$ typical at $10 \mathrm{Gbps}$, leading to optical link budgets of up to $14 \mathrm{~dB}$. The units incorporate an integrated heating element for the VCSEL laser arrays to permit high-performance operation over the temperature range of $-40 \mathrm{C}$ to $+85 \mathrm{C}$, and can support data rates up to $14 \mathrm{Gbps}$ per lane. The $100 \mathrm{~Gb}$ Ethernet standard is also supported using the $25.78 \mathrm{Gbps}-$ per-lane version of these parts, in the same form-factor.
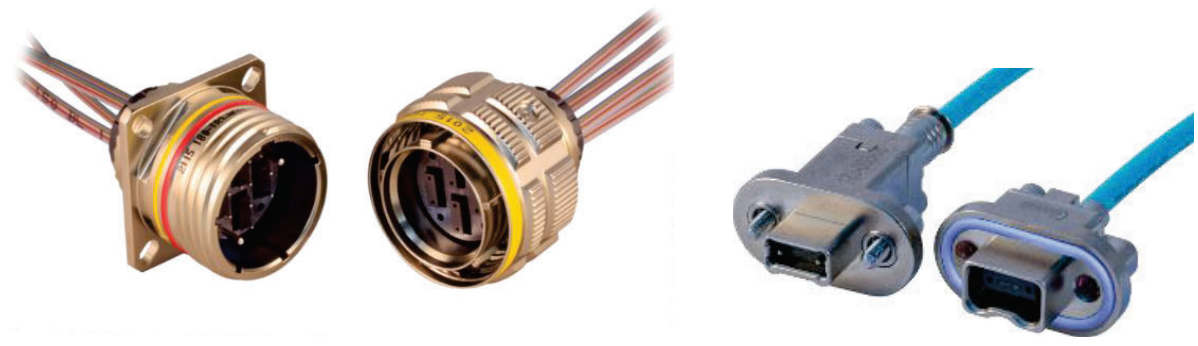

Figure 1: Rugged MT connectors: circular D38999 (left) and micro-D (right). 

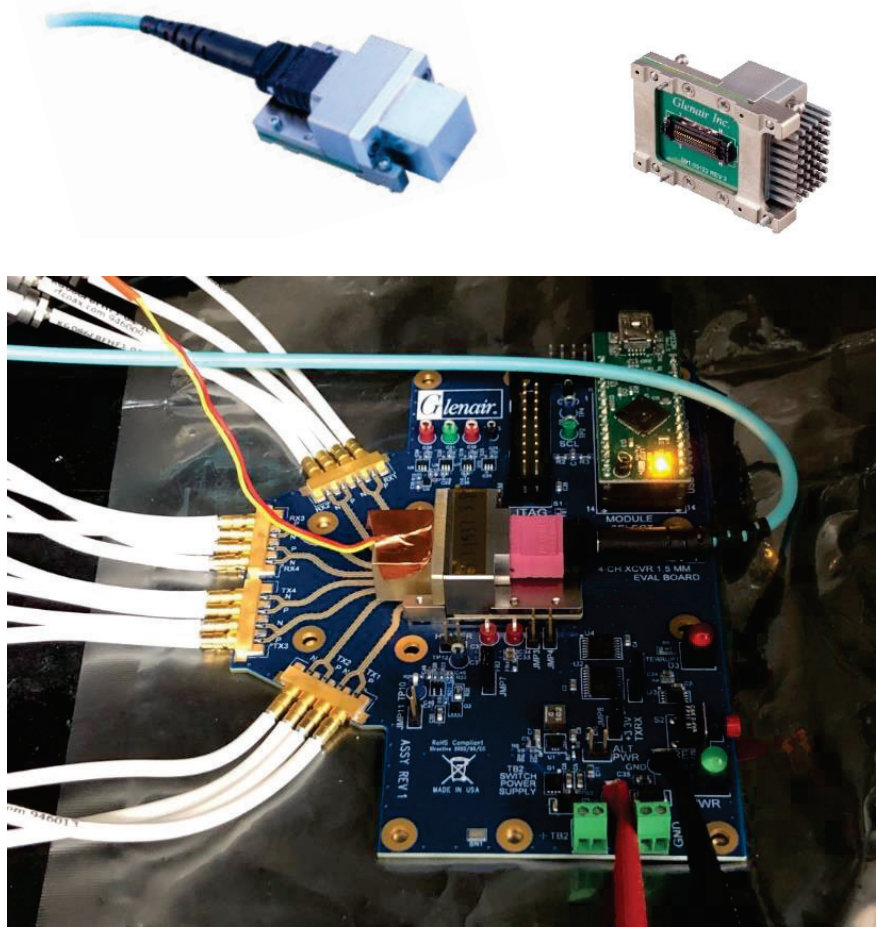

Figure 2: Aerospace-grade parallel optical transceiver: top view with MTP optical cable inserted and conduction-cooling heatsink (upper left), bottom view showing high-speed electrical connector interface and convention-cooling heatsink (upper right), transceiver mounted to 25 Gbps evaluation board (lower).

The transceivers utilize an MTP optical connector that is specially modified to provide enhanced vibration and shock survivability. The parts interface with the host board via a high-speed board-to-board connector with proven performance. These parts have high reliability in excess of $1 \mathrm{M}$ hours at $50 \mathrm{C}$ based on FIT rate data for the lasers, photodiodes and other integrated circuits contained in the part.

The transceivers have been subjected to operating shock and vibration levels per MIL-STD- 883 of $650 \mathrm{G} 1 \mathrm{msec}$ shock, 10 pulses in each axis and 46 Grms random vibration, 2 hours per axis. The hermetically-sealed units also pass MILSTD-883 helium leak rate testing as well as 10-day humidity testing with thermal cycling. The units were exposed to all of these tests while operating and bit errors were monitored at $5 \mathrm{Gbps}$. No errors were detected during any of these exposures. Heavy ion radiation testing was conducted on these devices up to $64 \mathrm{MeV}$ LET, with no unrecoverable events.

Extension of the bit rate to $25.78 \mathrm{Gbps}$ per lane has been completed and demonstrated over $-40 \mathrm{C}$ to $+100 \mathrm{C}$ case temperature. At $+100 \mathrm{C}$, the unit consumes $598 \mathrm{~mA}$ at $3.3 \mathrm{~V}$; at $-40 \mathrm{C}$ case temperature, the unit consumes $508 \mathrm{~mA}$ (heater off). The eye diagrams for $25.78 \mathrm{Gbps}$ at $100 \mathrm{C}$ case temperature are shown in Figure 3, which shows worst-case performance. Eye mask margins are improved at lower temperatures. Typical data associated with this performance is summarized in Table 1. 

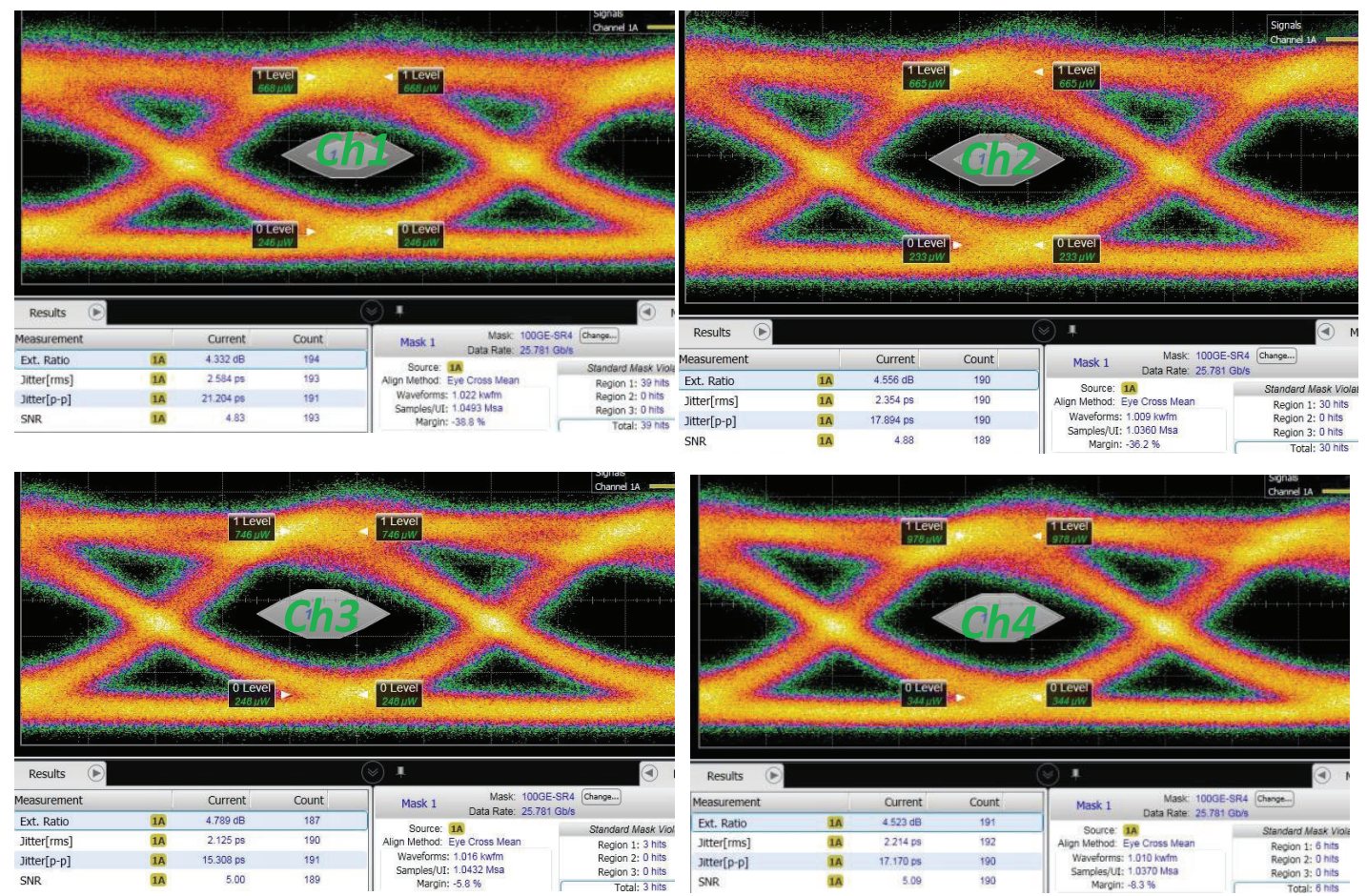

Figure 3. Transmitter eye diagrams for Glenair parallel optical transceiver operating at 25.78 Gbps data rate at $100 \mathrm{C}$ case temperature.

\begin{tabular}{|l|l|l|l|l|}
\hline Data/Ch. & Ch1 & Ch2 & Ch3 & Ch4 \\
\hline AOP (dBm) & 2.1 & 2.2 & 2.0 & 2.3 \\
\hline ER (dB) & 4.3 & 4.6 & 4.8 & 4.5 \\
\hline Jitter (ps rms $)$ & 2.6 & 2.4 & 2.1 & 2.2 \\
\hline SNR (dB) & 4.8 & 4.9 & 5.0 & 5.1 \\
\hline Margin (\%) & $\begin{array}{l}39 \\
\text { hits }\end{array}$ & $\begin{array}{l}30 \\
\text { hits }\end{array}$ & 3 hits & 6 hits \\
\hline
\end{tabular}

Table 1. Typical performance data for Glenair parallel optical transceiver at $25.78 \mathrm{Gbps}$ at 100C case temperature. 


\section{TRANSCEIVERS FOR FSO SYSTEMS}

DWDM transceivers for space applications were developed to support the ITU C-band grid frequencies that can be amplified by Erbium and Erbium-Ytterbium optical amplifiers. The transceiver, shown in Figure 4, is a printed-circuitboard mountable module that conforms electrically and optically to the SFP+ standard. It uses a transmitter optical subassembly (TOSA) and driver amplifier/controller and thermoelectric cooler (TEC) controller ICs. The TOSA uses an InGaAsP electro-absorption modulator and distributed-feedback (DFB) laser diode integrated photonic circuit chip mounted to a thermo-electrical cooler (TEC), and coupled to optical fiber through an optical isolator.

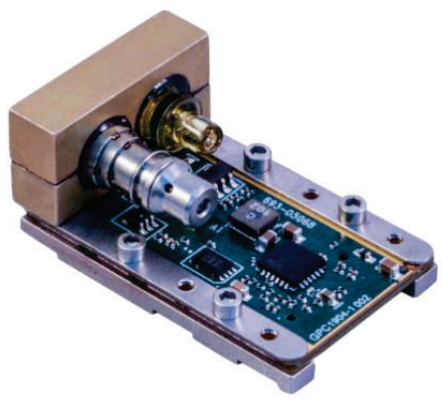

Figure 4. Glenair DWDM transceiver module. Optical fibers not shown for clarity.

The receiver section uses a hermetic receiver optical subassembly (ROSA) incorporating an InGaAs PIN photodiode integrated with a transimpedance amplifier IC, and an optional limiting amplifier. The firmware to run the controller IC resides in a Si CMOS EEPROM. The EEPROM can be removed and the firmware loaded at startup from an external processor via an $\mathrm{I} 2 \mathrm{C}$ serial interface to improve radiation tolerance. The transceiver controller IC is addressable via I2C interface to monitor and control the unit. A Windows-based graphical user interface is available to control the unit.

The typical output power of the transmit section is $0 \mathrm{dBm}$, and the unit can be provided with either a single-mode or a polarization maintaining fiber optic output fiber assembly. The transmitter zero-crossing can be adjusted via I2C to precompensate for the effects of optical amplification in an FSO link. The transmitter wavelength is maintained to $+/-100$ $\mathrm{ppm}$, and this may also be adjusted via I2C to compensate for Doppler shift due to platform motion, or to align with an optical filter to reduce amplified spontaneous emission (ASE) in the receiver. Typical extinction ratio at $11.3 \mathrm{Gbps}$ is $>9$ $\mathrm{dB}$. The operating temperature range is tested in the factory from $-40 \mathrm{C}$ to $+85 \mathrm{C}$.

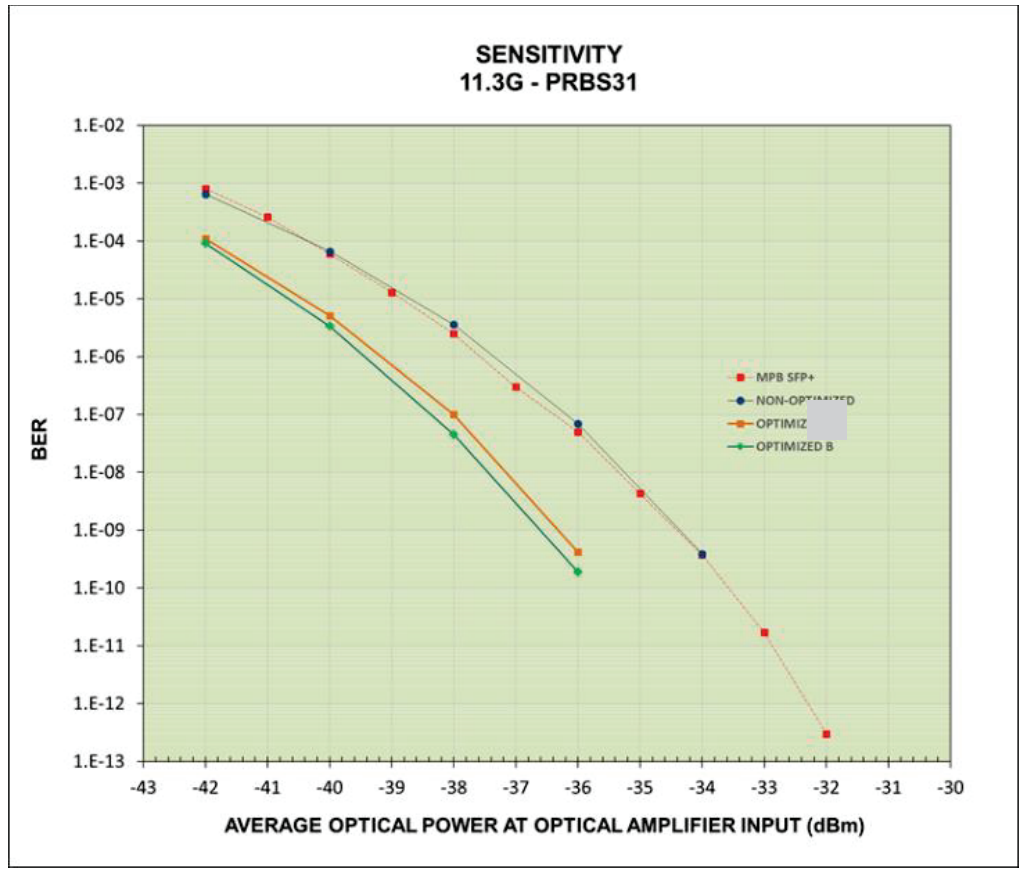

Figure 5. BER vs optical input power to low-noise optical amplifier for various configurations. 
BER curves in an optically-amplified system are illustrated in Figure 5 for the case of a commercial DWDM SFP+ using an EA modulator, the non-optimized Glenair DWDM EAM transceiver, and for optimized settings. By adjusting the zero-crossing and decision threshold appropriately, approximately $3 \mathrm{~dB}$ of link margin may be obtained, compared to a standard SFP+ transceiver.

\section{OPTICAL AMPLIFIERS}

Optical amplifiers suitable for space vacuum applications with up to $5 \mathrm{~W}$ of optical output power were developed. The amplifier design includes a 5W two-stage Erbium/Erbium-Ytterbium-doped-fiber booster amplifier, and an Erbiumdoped-fiber low-noise amplifier. The units utilize uncooled 980nm and 940nm pump lasers with hermetic packaging. All high-power optical components are heatsunk to a common aluminum baseplate, and long-term operation in thermalvacuum conditions with ambient pressure $<5 \mathrm{E}-6$ torr for $\sim 1000$ hours has been demonstrated from $-5 \mathrm{C}$ to $+60 \mathrm{C}$ baseplate temperature. All pump power control circuitry is managed by a microprocessor that is available in a radiationtolerant version. The unit is available in single-mode and polarization-maintaining fiber versions. One physical implementation of the $5 \mathrm{~W}$ booster+LNA unit and a $1 \mathrm{U}$ cubesat booster with $1 \mathrm{~W}$ output power are shown in Figure 6. Other configurations can be accommodated.
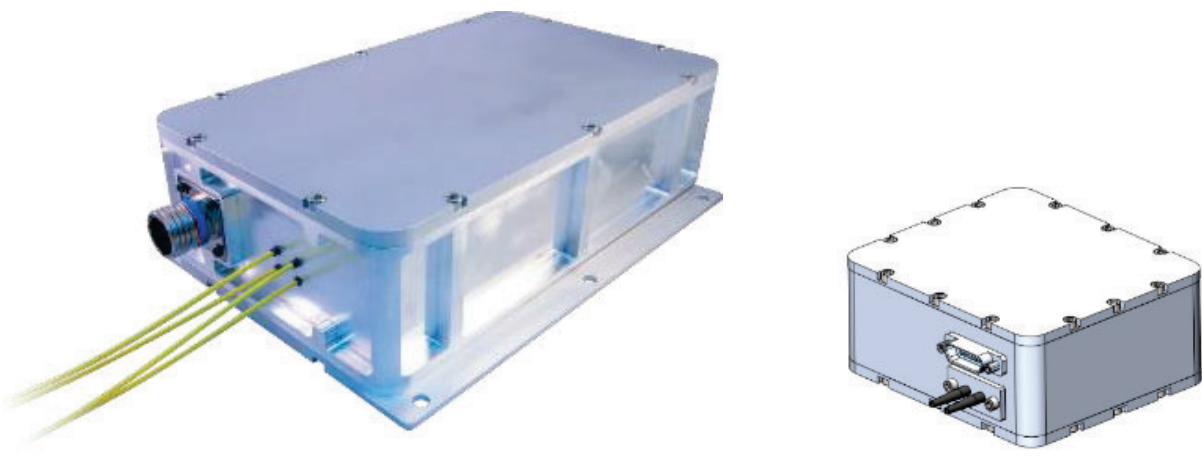

Figure 6. Optical amplifier types: $5 \mathrm{~W}$ booster + LNA (left) and $1 \mathrm{~W}$ booster only for $1 \mathrm{U}$ cubesat (right, in development).

\section{SUMMARY AND CONCLUSIONS}

We presented transceiver and optical amplifier modules suitable for on-board and inter-satellite FSO spacecraft high speed digital datalinks. We discussed the results of $25 \mathrm{Gbps}$ parallel optical transceiver, 11.3 Gbps DWDM FSO transceivers, and optical amplifier modules. We subjected them to various tests, including thermal cycling, high 
vibration and shock, and radiation, and found them to have excellent performance. Testing to date indicates that these parts can satisfy the environmental requirements of spaceflight applications. Further testing and product development is ongoing, and results will be reported in future publications.

\section{REFERENCES}

[1] R.T. Logan Jr. and D. Basuita, Proc. of International Conference on Space Optics, Crete, 2018.

[2] R. T. Logan Jr. and D. Basuita, Proc. of International Conference on Space Optics, Biarritz, 2016.

[3] R. T. Logan Jr., Proc. of International SpaceWire Conference, Yokohama, October 2016. 